\title{
Cambios en la significación de su propio cuerpo en mujeres adultas obesas que se han sometido a cirugías bariátricas
}

\author{
Changes in the significance of their own \\ bodies in obese women who have \\ undergone bariatric surgery
}

\begin{abstract}
This paper explores how young obese women view their own body's change after undergoing bariatric surgery. As a qualitative study, the effort draws upon personal interviews which were analyzed according to the concepts of the selected theoretical framework. The sample consisted of four female interviewees between the ages of 18 and 35. The results of the study indicate that following surgery women experience not only physical changes, but also changes in their personal understanding of their bodies. In addition to these changes, there was an increase in self-care behaviors, an improvement in the relationship between the body and social environment, as well as a rise in self- esteem. This paper concludes that bariatric surgery contributes to the unification of a previously fragmented perception of mind and body. In addition, it shows that the presence of psychological therapy during the post operational period is valued by the interviewees and seen as a way to prevent future ruptures in the psycho-soma integration.
\end{abstract}

Key words: body; obesity; significance, bariatric surgeries.

\section{INTRODUCCIÓN}

El ideal de belleza de la mujer ha ido variando con el paso del tiempo. Los cánones de belleza actual promueven la imagen de una mujer más delgada comparada con décadas anteriores. Pero en la realidad no toda mujer alcanza este arquetipo ideal del cuerpo, por lo que surge la pregunta: ¿qué sucede con las mujeres que no cumplen estos estándares? ¿Cómo vivencian su propio cuerpo dentro de una cultura que construye un ideal de cuerpo basado en la delgadez? El cuerpo, desde la perspectiva de Françoise Doltó, es definido como un intermediario entre el mundo y el sujeto (1), por lo tanto, es un mediador entre la mujer, su contexto y la cultura en la cual se desarrolla. La imagen en nuestra sociedad actual es todo, si no te ven no existes, lo que lleva a hablar de la tiranía de la visibilidad (2).

La obesidad es definida como "un síndrome de etiopatogenia multifactorial caracterizado por un aumento del tejido graso" (3). Actualmente, es la enfermedad nutricional de mayor prevalencia en la población mundial menor de 20 años, y es considerada "la epidemia del siglo XXI", ya que es una enfermedad que es padecida por gran parte de la población (3).

Esta enfermedad se asocia a otras enfermedades médi-
Daniela Carrasco $D$. Daniela Appelgren D. Juan Carlos Núñez $M$. Constanza Schlanbusch $M$.

Facultad de Psicología, Universidad Diego Portales. Santiago, Chile.

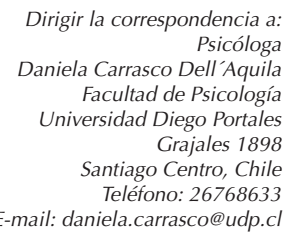

Este trabajo fue recibido el 3 de Septiembre de 2013 y aceptado para ser publicado el 18 de Enero de 2014.

cas graves como problemas cardiovasculares, hipertensión, dislipidemias, diabetes, entre otros, las que disminuyen las expectativas de vida de los sujetos que las padecen (4).

Según la Encuesta Nacional de Salud 2010- 2011(5), un $67 \%$ de la población presenta exceso de peso, y si observamos cómo se distribuye este porcentaje por género, nos encontramos con que tanto en obesidad como en obesidad mórbida, es mucho más alto en mujeres: 30,7 en mujeres vs 19,2 en hombres en obesidad, y 3,34 en mujeres vs 1,26 en hombres en lo que se refiere a obesidad mórbida.

El porcentaje nacional de obesidad, indicado por esta encuesta (5) nos muestra que del total de $25,1 \%$, el $30,7 \%$ corresponda al género femenino y el $19,2 \%$ al masculino.

Frente a esta realidad han surgido una gran variedad de tratamientos, sin embargo la efectividad de estos aunque sean multidisciplinarios no son efectivos en pacientes obesos mórbidos (efectividad menor al 10\%) y muchos recuperan el peso a largo plazo, por lo que los resultados son decepcionantes e insatisfactorios, lo que provoca que estos pacientes opten por intervenciones quirúrgicas. Esta opción es más efectiva y muestra resultados casi inmediatos. En los últimos 10 años, la alternativa quirúrgica ha mostrado ser la opción más efectiva 
(6). En Chile, las cirugías mas realizadas para el tratamiento de la obesidad son la banda gástrica ajustable (realizada en un $40 \%$ de los casos) y el bypass gástrico laparoscópico $(30 \%$ de los casos) (6).

El presente estudio abordó las percepciones y significaciones sobre el propio cuerpo que poseen las mujeres adultas con obesidad mórbida, que se han sometido a cirugías bariátricas.

La decisión de entrevistar exclusivamente a mujeres se debe a los porcentajes antes indicados, que señalan que son ellas las que tienen mayor prevalencia de obesidad mórbida en Chile (5).

La mayoría de los autores, están de acuerdo en que la cirugía debería reservarse para pacientes con obesidad mórbida, en los que todas las demás terapias han fracasado y que sufren complicaciones metabólicas importantes. Los criterios más utilizados, independientes del sexo, son:

a) Presentar un IMC > 40 ó > 35 con comorbilidades.

b) Que la obesidad grave esté presente desde hace más de 5 años.

c) Que no exista historia de alcoholismo y otras drogodependencias o enfermedad psiquiátrica grave.

d) Que los pacientes tengan entre 18 y 60 años.

e) Que exista una adecuada comprensión de las alteraciones producidas por la intervención y una buena posibilidad de adhesión a la estrategia terapéutica propuesta (7).

La obesidad, además de tener una etiología fisiológica y de alterar el cuerpo físicamente, tiene factores etiológicos psicógenos, afectando directamente a la psique del sujeto que la padece. (8) Si bien esta enfermedad no se encuentra en el DSM-IV (Diagnostic and Stadistical Manual of Mental Disorders) como un trastorno primeramente psiquiátrico, es considerada una condición médica multideterminada etiopatogénicamente y con variadas manifestaciones clínicas que en ocasiones tienen competencias psicopatológicas (9).

La relación psicopatología-obesidad se establece en tres niveles: (10).

1. El primer nivel se relaciona con el factor psicógeno observable en algunas obesidades, el cual si no es valorado, no podría realizarse un tratamiento terapéutico adecuado y eficaz.

2. El segundo nivel refiere a alteraciones psicopatológicas reactivas desarrolladas por un alto porcentaje de los pacientes. Éstas generalmente no son graves y sus intensidades no son proporcionales a la gravedad de la obesidad.

3. El último nivel tiene que ver con las reacciones distímicas producto de tratamientos dietéticos y/o farmacológicos fallidos realizados por algunos pacientes obesos (10).

Es por esto que las intervenciones deben ir acompañadas de un apoyo interdisciplinario de profesionales, asistencia psicológica para la aceptación del nuevo cuerpo y apoyo de un experto en nutrición que permita reeducar al paciente a través de la búsqueda de nuevos hábitos alimentarios, todo esto para lograr un desarrollo de un nuevo estilo de vida más saludable (11).

En este sentido resulta interesante abordar los cambios en la percepción y significación del propio cuerpo en mujeres que se han sometido a estas intervenciones, con el fin de aportar en el proceso de aceptación del nuevo cuerpo, facilitando el período post operatorio. Aportar a dicho proceso post operatorio desde la concepción de otro tipo de cuerpo, un cuerpo distinto al de la medicina. No es sólo a un cuerpo anatómico, fisiológico, sino que hablamos de un cuerpo erógeno, el cual es recorrido por una energía vital que está en directa relación con la historia del sujeto. Es un cuerpo que se construye, se conquista como resultado de la inscripción que dicha historia hace en él, surgiendo un cuerpo erogenizado que se diferencia cualitativamente de un simple pedazo de carne (1).

Otro aspecto importante que se abordó son los resultados de estudios que dan cuenta de un auto concepto negativo en personas obesas, especialmente en mujeres (6), y otros que evidencian que la baja de peso producto de las cirugías bariátricas tiene buenos resultados psicosociales; mejoras en la calidad de vida de los pacientes, en su salud, y en la socialización, disminuyendo los síntomas depresivos y de ansiedad (6). Respecto a la evaluación del autoestima en los estudios realizados por estos autores, se evidenció que personas que se sometieron a la cirugía bariátrica presentan una mejora significativa en la autovaloración y en la autoestima.

El sujeto, a medida que crece y se desarrolla, se va apropiando y adueñando de este cuerpo, lo va conociendo y haciéndose consciente de él (12). Esta construcción del cuerpo está mediada por las fantasías tanto como por las experiencias de vida.

El objetivo de esta investigación fue determinar la presencia de cambios en la significación de sus cuerpos, en mujeres adultas obesas mórbidas, que se sometieron a cirugías bariátricas.

\section{SUJETOS Y MÉTODO}

Se realizó una investigación cualitativa que buscó comprender las significaciones, perspectivas y opiniones subjetivas de mujeres adultas obesas que se sometieron a cirugías bariátricas (bypass gástrico o banda gástrica ajustable). Se partió de la premisa de que el grupo estudiado es subjetivo y relativo, por lo que se entendió y se profundizó el discurso desde sus propias significaciones (13). Consistió en un estudio descriptivo que buscó detallar las características de la significación que le dieron las mujeres entrevistadas a su propio cuerpo luego de la intervención. El estudio fue abordado desde la teoría psicoanalítica que nos permitió entender el sujeto como resultado de la unión psique-soma (12).

\section{Muestra}

La población o grupo de estudio a partir del cual se realizó la investigación corresponde a cuatro mujeres chilenas adultas previamente diagnosticadas con obesidad, cuyas edades fluctuaban entre 18 y 35 años, todas habitantes de la Región Metropolitana de Santiago y sometidas a cirugías bariátricas (bypass gástrico o banda gástrica). Tres de las entrevistadas terminaron su educación superior universitaria y la más joven se encuentra cursándola. Sólo una de las entrevistadas estaba emparejada al momento de la operación. La intervenciones quirúrgicas fueron realizadas entre los años 2003 y 2012.

\section{Instrumentos}

El instrumento utilizado fue una entrevista semiestructurada que contemplaba temas específicos a indagar, destinadas a recopilar información sobre la fecha y tipo de cirugía, los problemas médicos asociados, el motivo de la operación, las fantasías y miedos para con ésta, el autoestima, el rol del apoyo psicológico en este proceso, la relación con su propio cuerpo, los cambios que se produjeron con la cirugía (físicos y psíquicos), los hábitos alimentarios y de ejercicio, y las transformaciones en la significación del propio cuerpo después de la cirugía.

El orden y la formulación de las preguntas variaron en cada entrevista, y los temas que surgieron también fueron interrogados, como la asociación que surgió entre subida 
de peso y embarazo fue rescatado del discurso directo de las entrevistadas quienes señalaban: "yo subí con los embarazos, tengo 3 hijos hombres y con cada uno subí, con el primero bajé completo, no tuve ningún problema; con el segundo me quedé, no sé, como con 10 (kilos) arriba; y con el tercero... y con el tercero, ya, no sé, debo haber subido como 30 kilos y bajé 15 con dieta y todo, y después no pude seguir bajando.

\section{PROCEDIMIENTO}

Se realizó una entrevista individual a cada una de las participantes la que fue llevada a cabo por dos de las investigadoras. La duración de cada una de ellas fue de una hora y media, aproximadamente. Estas fueron grabadas en formato de audio.

Luego de transcritas las entrevistas, se analizaron los relatos de cada una de las mujeres entrevistadas, determinando de esta forma distintas categorías comunes entre las entrevistas a partir de los fenómenos encontrados a través de un análisis de contenido.

Posteriormente los resultados fueron analizados e interpretados a partir del marco teórico escogido. No se hizo ningún tipo de seguimiento a las entrevistadas, ya que el interés se enmarcó en recopilar la información requerida en cualquier instancia posterior a la intervención quirúrgica, por lo que no fue necesario hacer un seguimiento a la muestra para los fines de la investigación.

\section{ANÁLISIS Y DISCUSIÓN DE RESULTADOS}

Específicamente el estudio arrojó la siguiente información en relación a las variables estudiadas:

1.- PROBLEMAS MEDICOS ASOCIADOS. La información obtenida se contrapone con lo que se esperó en un principio de la investigación: las enfermedades asociadas a la obesidad no fueron factores relevantes de motivación para someterse a las cirugías.

En contraposición con lo que se habría esperado en un principio de la investigación, las enfermedades asociadas a la obesidad no fueron factores relevantes de motivación para someterse a las cirugías. Sólo una de las entrevistadas manifestó tener mayores molestias y problemas físicos asociados a su obesidad, mientras que las otras tres afirmaron que no padecían ninguna enfermedad física producto de la obesidad que les produjera mayor malestar, y si los tenían, se habrían mantenido luego de la operación, como por ejemplo el colesterol alto (Florencia). Más que el padecer una enfermedad asociada a la obesidad en ese minuto, era el miedo a padecerla en el futuro, el tener el conocimiento de cuán dañino para su salud era el ser obesas.

2.- MOTIVO DE LA OPERACIÓN. Las motivaciones de las pacientes para llevar a cabo la cirugía eran intrínsecos y extrínsecos; se destacan la necesidad de sentirse más valoradas y aceptadas socialmente, aumentar su autoestima e insatisfacción con su imagen, lo que afectaba todos los aspectos de sus vidas, en este sentido, Andrea dice: "[...] el sentir que el resto de la gente te mira así como pobrecita porque no era así, era tan linda [...]"Si bien las entrevistadas señalaron su preocupación por su estado de salud futura, cabe destacar que ninguna de las cirugías fueron indicadas por problemas metabólicos. En este sentido una de las motivaciones por las cuales las entrevistadas tomaron finalmente la decisión de someterse a estas intervenciones, se encuentran la sensación de frustración que se genera luego de tantos intentos fallidos por bajar de peso. Las entrevistadas refieren que a pesar de los esfuerzos que hacían, no lograban bajar de peso de ma- nera significativa, lo que generaba frustración y sentimientos de impotencia ante la situación: "[...] Es frustrante, porque hacis todo el esfuerzo para bajar, y no bajai [...]" (Andrea), "es típico el efecto rebote de las dietas porque pasai semanas sin comer nada y de repente, se te hace como una droga, y te lo comis todo y subis más de lo que habiai bajado antes [...]"

Además de lo anterior, se pueden apreciar motivaciones de carácter externo como lo son encontrar talla de ropa en las tiendas y tener la aceptación de gente externa como familiares y/o amigos, sobre lo cual Florencia comenta que no estaba muy convencida de operarse, "[...] pero mi papá insistió tanto y tanto tiempo que me convenció."

Otro factor nuevo que no estaba contemplado y que surgió a partir de las entrevistas fue el alza de peso producto de los embarazos de las mujeres. En dos de los cuatro pacientes subieron de peso más significativamente durante y después de sus embarazos. Ambas expresaron haber aumentado considerablemente de peso durante y después del embarazo, y que a pesar de los intentos, no lograron volver a su peso inicial: ninguna de ellas logró reconocerse en su cuerpo, sintiéndolo ajeno. Esto nos indica que la significación del propio cuerpo sería producto de la historia de vida de los sujetos, de los hechos importantes que han marcado su desarrollo. Ejemplifica también cómo estos hechos quedan inscritos tanto en la psique como en el cuerpo mismo (embarazos, relaciones amorosas conflictivas y cirugías bariátricas) (1).

3.- FANTASÍAS Y MIEDOS SOBRE LA OPERACIÓN: En cuanto a las fantasías, todas se relacionan con un aumento en la autoestima cumpliendo metas para sentirse más felices. Las fantasías y expectativas son también influenciadas por el resto ya que ellos también fantasean y tienen expectativas acerca de esta cirugía. Andrea refiere a sentirse bonita, lo que da cuenta que quería mejorar su autoestima y poder usar la ropa de antes. Soledad nunca se hizo muchas expectativas o por lo menos no se refiere a ellas. Florencia quería volver a pesar lo mismo que antes de su embarazo y se infiere que su familia tenía altas expectativas ya que ella no quería que la molestaran más. Por último, Javiera, da cuenta que sus expectativas se relacionaban con ser sana, y fantaseaba con aceptación social.

Lo anterior queda reflejado en lo que dice Andrea en relación a sus propias expectativas y las del resto, asegurando que quería: "[...] Recuperar mi peso, o sea recuperar mi ropa [...]" " [...] Sentirme bien [...]" "[...] el sentirte, sentir, sentirse bonita cachai?, sentirse bonita [...]" "[...] hay ene expectativa (del resto) y como que están esperando, porque como el primer mes bajai tanto, esperan que la cuestión siga así [...]"

En todos estos relatos se muestran expectativas y fantasías específicas de las entrevistadas, que apuntan a un mismo aspecto importante de la vida, ponerse metas y lograr alcanzarlas para lograr sentirse más felices, realizadas y conformes consigo mismas.

4.- AUTOESTIMA. La forma de relacionarse con el mundo también cambió después de la cirugía, al igual que el modo en que el mundo se relaciona con ellas. Estos cambios evidencian una mejora en la autoestima de todas las entrevistadas, ya que al sentirse más aceptadas, desarrollaron también una aceptación para con ellas mismas. Lo anterior permite hipotetizar que existe una correlación significativa entre el aumento de autoestima y un cambio positivo en la forma de relacionarse con el mundo y con su propio cuerpo. Tres de las cuatro entrevistadas reconocen explícitamente que tenía un autoestima muy baja antes de someterse a la operación. Soledad da cuenta de esto a través de su forma de vestir, Andrea y Javiera 
reconociendo derechamente que es un período en el que no se querían y por último Florencia sin hacer una evaluación negativa de su autoestima antes de la cirugía, reconoce que después sí se sentía más bonita y que se comenzó a arreglar más al igual que las demás entrevistadas.

Las citas rescatadas muestran la correlación existente entre baja de peso y autoestima; y además reconoce que estas mujeres tenían una autoestima baja cuando eran gordas. Soledad (34) se refiere al período previo de la operación señalando: "[...] como que en ese período uno está como ausente, uno se abandona [...]" "[...] Yo me di cuenta que uno no se quiere, y es eso, como que se auto castiga [...]" "[...] todo el rato es estoy gorda, estoy gorda, estoy gorda, estoy fea, estoy gorda, soy tonta; no se po [...]" Y se refiere al período actual diciendo: "[...]soy más feliz, pero soy la misma, he crecido, soy más segura [...]"

Esta última cita refleja una realidad común a todas las entrevistas; en todas existe un cambio positivo en su autoestima.

5.- ROL DEL APOYO PSICOLOGICO. En cuanto al rol que cumple un psicólogo o psiquiatra en este largo proceso de aceptación, adaptación, educación y relación con este nuevo cuerpo, todas valoran su importancia. Si bien sólo dos de las entrevistadas tuvieron un apoyo psicoterapéutico luego de la intervención, las otras dos no se cierran a esta posibilidad, ya que reconocen que es de gran ayuda, que habrían momentos en los que sería muy útil y que el apoyo haría más llevadera la readaptación al mundo. A través de la terapia, lograron entender que belleza no es sinónimo de extrema delgadez, sino que es sinónimo de salud.

En todas las entrevistas surgió el concepto de ansiedad como eje importante, ya que muchas veces se la asocia con la ingesta de comida; ansiedad que después de la operación, no se tradujo en ingesta de comida -dada la aprehensión e nuevos hábitos de alimentación- si no que ahora es canalizada a través de la práctica de deporte, y en uno de los casos, es tratada con medicamentos.

Ambas entrevistadas acudieron a psicoterapias antes de la operación: "[...] yo tuve que hacer todo un tratamiento antes de operarme [...]" "[...] De hecho yo sigo yendo al psiquiatra [...]" "[...] La cabeza es un proceso mucho más largo [...] pero cuesta mucho más el proceso [...]" (Soledad). Soledad afirma que a través de la terapia, con ayuda de psicólogos y psiquiatras, logró entender que belleza no es sinónimo de extrema delgadez ni mucho menos de anorexia, sino que, en realidad, la belleza es sinónimo de salud. Y continúa diciendo que hoy en día, luego de aproximadamente diez años de su operación, sigue yendo al psiquiatra, manifestando que es un proceso largo y complejo, es un cambio más difícil de asimilar y es de gran ayuda el acompañamiento psicológico para lograr y mantener el equilibrio.

Siguiendo con el mismo tema, Javiera afirma: "Estuve a punto de que no me operaran por temas psicológicos [...]" "[...] un tema demasiado importante en la operación es ir al psicólogo y al psiquiatra. Yo voy dos veces a la semana o tres veces a la semana al psicólogo." Afirma que siempre se sintió acompañada por este equipo, el cual siempre estuvo a su disposición en caso de tener cualquier duda o inseguridad con respecto a la cirugía: "[...] es un equipo entero multidisciplinar que iba conmigo. Siempre me sentí acompañada por ese equipo."

De esta forma se destaca el importante rol que cumple el psicólogo en este complejo proceso, abriendo la posibilidad de prestar servicio en conjunto con otras disciplinas, ampliando de esta forma aún más el campo trabajo de la psicología.
6.- RELACION CON SU PROPIO CUERPO. Los cambios se evidencian tanto en la forma en que se refieren ahora a su cuerpo como en actividades concretas que actualmente realizan, la forma en que cuidan ahora de sus cuerpos, tomando conciencia de cuánto y cómo afecta la concepción de ellos en su psique y viceversa. Afirman que actualmente sus cuerpos juegan un rol fundamental en sus vidas y son objeto de especial atención.

Las respuestas de las entrevistadas reflejan que sí existen cambios en la forma en que estas mujeres se relacionan con su cuerpo, siendo Soledad quien dice tajantemente: " [...] tu relación con tu cuerpo empieza a ser otra, empiezas a conocerte completamente [...]"; De ellas surge una reflexión que hace referencia a una nueva forma de mantener contacto con su cuerpo, afirmando que antes no habrían tenido una relación muy estrecha con su cuerpo, es decir, no habrían sido tan conscientes de él: "Antes no tomaba tanta conciencia de la relación que tenía con él, o sea, de si lo cuidaba o no, y si lo hacía, de cómo lo hacía." (Andrea). Sin embargo luego de la intervención se habrían hecho más conscientes de éste, sobre lo cual Andrea dice: "[...] no sé si ahora soy la más consciente del mundo, pero me preocupo, lo cuido, aparte que estoy obligada, o si no lo noto al tiro, me avisa que algo hice mal."

Todas coinciden en la valoración de este cambio, valorándolo como un cambio totalmente positivo y que les habría traído buenas consecuencias en múltiples ámbitos de sus vidas. También habrían logrado un mayor dominio y control sobre su cuerpo y sobre sí mismas, características que serían consideradas como necesarias para mantener el éxito de la cirugía en el tiempo. Esto daría cuenta no sólo de cambios en la relación con su cuerpo, sino de cambios que trascenderían a varios aspectos de sus vidas y que marcarían un antes y un después en su línea de vida producto de la intervención quirúrgica a la que se sometieron. Lo anterior se evidencia de forma concreta y real en aspectos concernientes al cuidado del cuerpo, como el ejercicio físico, los hábitos alimentarios y la preocupación por cuestiones más bien estéticas, aspectos que se presentan a continuación.

7.- CAMBIOS EN EL MODO DE RELACIONARSE CON EL MUNDO. Todas, excepto Florencia, reconocen de forma explícita que ha cambiado el modo en que se relacionan con su contexto. Cada una de acuerdo al periodo de vida que está viviendo. Andrea pone énfasis en la relación con sus hijos, Soledad en relación a los hombres y Javiera en relación a su aceptación social. Estas tres mujeres dan cuentan de un cambio positivo en diferentes áreas pero que apuntan a lo mismo.

Este cambio se ve explicitado en lo que dice Andrea: "[...] el sentir que el resto de la gente te mira así como pobrecita porque no era así, era tan linda [...]" "[...] yo no iba a estar bailando ni que me anden mirando [...]"

Estos cambios se ven más manifiestamente en Javiera quien asegura que al bajar de peso "[...] fue una aceptación al tiro en el grupo, como el ver que soy una más y que paso piola $[\ldots] "$

8.-CAMBIOS EN LOS HABITOS ALIMENTARIOS Y DE EJERCICIO. En todos los relatos de las mujeres se muestran cambios en cuanto al cuidado de sus cuerpos. Tres entrevistadas comenzaron a realizar actividad física de manera frecuente luego de la cirugía para mejorar y mantener su figura y musculatura. Los hábitos alimentarios cambiaron en todas ya sea por voluntad propia o exclusivamente debido a las restricciones correspondientes a la operación, ordenando y organizando las comidas, siguiendo un régimen estricto y disminuyendo 
la cantidad de ingesta de alimentos.

En la mayoría de ellas se observaron cambios en el cuidado más estético del cuerpo, lo que se evidenció en la inversión de dinero en cremas corporales, en ropa nueva y bonita e incluso en la compra de maquillaje y cursos de maquillaje para aprender a usar de la mejor forma las nuevas adquisiciones. Después de la cirugía, todas manifiestaron mayor control y autocuidado, existiendo cambios en relación a la actividad física, la alimentación y el cuidado estético.

Esto se vió reflejado en los nuevos hábitos alimentarios que adoptaron: comer bien, en menor cantidad, más lento y darse cuenta de su nueva y real capacidad en lo que a alimentación respecta. En este sentido Florencia, a pesar de ser quien menos respeta los nuevos hábitos alimenticios, igualmente da cuenta de este cambio, que se refleja en lo siguiente: "[...] con lo de la dieta, me salgo súper harto pero si como no sépo un pedacito de pan que no puedo comer, quedo tan llena que después no me da hambre en demasiado rato entonces no como. Muchas cosas igual ahora me dan asco y antes era seca, como las frituras y cosas más cochinas [...]".

También se puede observar en la forma en que cuidan sus cuerpos, dado que antes de la cirugía, ninguna (o casi ninguna) de las entrevistadas cuidaba mucho su cuerpo; sin embargo a partir de la operación comenzaron a preocuparse más por él aplicándose distintos productos (variadas cremas humectantes, para las estrías o para las cicatrices), tomando vitaminas $y / 0$ realizando algún tipo de actividad física.

9.- CAMBIOS EN LA SIGNIFICACIÓN DEL CUERPO. se observó que sí existen cambios en la significación propia del cuerpo luego de la cirugía bariátrica a la que se sometieron. Entendiendo el cuerpo no sólo como un cuerpo físico, sino también erógeno (12), el cual se conquista y construye en el tiempo y que guarda relación con la historia particular de cada individuo (1): se evidencia además de un cambio físico, un cambio en la significación del propio cuerpo. Se concluye que la cirugía bariátrica marca un hito en la vida de estas mujeres, a partir del cual comienza un proceso de reconquista, redescubrimiento y dominio del cuerpo.

Esta nueva significación habría tenido un efecto directo en la forma en que estas mujeres se relacionan con su cuerpo, y esto nos permite pensar en la importancia de entender la obesidad como un fenómeno psicosomático (12). O sea, comprender cómo se relaciona el sujeto con su cuerpo ya que en él van quedando inscritas las huellas de su historia, historia que también debería quedar inscrita en la psique. Si no se lleva a cabo dicho proceso, se produce una separación que puede explicar las conductas de abandono con ellas mismas, existiendo en todas incomodidad con su cuerpo y un descuido físico antes de la operación, quizás, como mecanismo de defensa para no asumir un cuerpo obeso. Una de ellas reconoce que "no se daba cuenta de lo gorda que llegó a estar". Como explica Winnicott, (12) en la enfermedad psicosómatica hay una separación entre la psique y el soma, lo que lleva a una escisión entre el cuidado físico y el cuidado de la psique.

Según el relato de las mujeres, tanto explícita como implícitamente se puede afirmar que efectivamente existen cambios en la significación de su propio cuerpo, sobre lo cual Florencia afirma: "[...] Como que ahora me doy cuenta de lo importante que es para mí el cuerpo, cosa que antes en verdad no era consciente, yo en verdad creo que me daba lo mismo [...]"

Estos cambios apuntarían a una mejora en su calidad de vida, en su percepción y valoración del cuerpo y en una mayor conscientización de esta unidad mente-cuerpo, cambios que se confirman en el discurso de Javiera, quien dice: "Ahora mi cuerpo es lo mejor que tengo, es la carta que tengo a jugar."

Se puede apreciar entonces un cambio drástico en el antes y en el después de la cirugía, siendo Javiera quien lo ratifica abiertamente diciendo: "[...] mi vida cambió radicalmente, el cambio fue demasiado bueno [...]

10.- SURGIMIENTO DE OTRAS TEMÁTICAS. Otro aspecto fueron las cicatrices en el cuerpo, no solo a causa de la intervención quirúrgica, sino también producto de la maternidad. En todas existe una preocupación, sin embargo, dicen llevarlo de buena forma ya que estas huellas les recuerda quiénes son y por qué son así. Las tres mujeres que se refirieron a este tema afirmaron que no les importaba tanto estas huellas físicas, ya que la ganancia de tenerlas era mayor: esto reafirma la posibilidad de que estas mujeres ahora se vivan más integradas, es decir, habitando sus cuerpos de los cuales han logrado apropiarse.

"[...] yo tengo mi guata con estrías, cachai, cosas que yo sé que van a quedar, pero no importa, o sea, no es tema [...] Si tengo que tener marcas y cicatrices, las tendré, y las llevaré orgullosa no más, si al final es la historia que también pasa por mi cuerpo, algún precio hay que pagar! [...] Además que yo desde el primer embarazo que quedé con ene estrías y todo, como que siento que mi mentalidad es distinta de otras amigas que han quedado embarazadas. Entonces es parte de mi proceso de ser mamá, de ser mujer, no tengo un tema con eso [...]" (Andrea).

Siguiendo la misma línea de las afirmaciones de Andrea, Soledad dice: "[...] me quedaron estrías [...] Aprendí a acostumbrarme porque la perfección no existe, o sea siempre va a haber algo que nos va a molestar de nuestro cuerpo [...]". Se induce que Soledad también asume sus cicatrices y marcas corporales como consecuencias aceptables de la cirugía, las cuales está dispuesta a llevar el resto de su vida. Aunque, si bien preferiría no tenerlas, tampoco le molestan de sobre manera, es decir, no son un tema importante en su vida, ya que al igual que Andrea y Javiera, afirma que si no las tuviera, no sería la persona que es ahora.

De esta forma expresa directamente que, al ser necesarias para lograr su meta, no le importa llevarlas durante toda la vida, sin dejar de cuidarlas para que no sean cicatrices mayores.

\section{CONCLUSIONES}

Luego de haber realizado esta investigación, se ha podido responder al objetivo general, o sea-describir los cambios en la significación de su propio cuerpo en mujeres adultas obesas entrevistadas, que se han sometido a cirugías bariátricas-.

Al releer todas las entrevistas y a partir de su contenido se podría afirmar que anteriormente no habría habido una mayor conciencia de sus cuerpos, y al intervenirlos quirúrgicamente se habrían hecho más conscientes de él, se habrían dado cuenta de que realmente está ahí y no solamente es un pedazo de carne que permite vivir, sino que es un aspecto fundamental el poder habitarlo, y por lo mismo merecedor de mayor cuidado y atención.

En relación a la motivación, encontramos que la cirugía fue la última opción después de varios intentos fallidos anteriores para bajar de peso y los cambios en la significación se vieron plasmados, luego de la cirugía, en la mejoría de la calidad de vida, y en la valoración positiva de su cuerpo.

Se concluye en la necesidad de que futuras investigaciones realizacen un seguimiento después de la cirugía bariátrica, para comprobar si estos cambios detectados en la significacion de sus cuerpos se mantiene en el tiempo. y/o incluir otras 
herramientas útiles para la recolección de información, como test proyectivos o gráficos, aplicados antes y después de la operación.

El desafío que queda por delante es seguir avanzando en las investigaciones multidisciplinarias, con el fin de lograr intervenciones más exitosas que comprendan la obesidad, no sólo desde una mirada médica que de cuenta de un cuerpo orgánico, sino también desde una perspectiva psicológica que permita dar cuenta de un cuerpo que es producto de la unidad psicosomática que cada persona es.

\section{RESUMEN}

El presente estudio cualitativo exploró los cambios en la significación otorgada al propio cuerpo, en mujeres adultas jóvenes y obesas, que se sometieron a cirugías bariátricas. Se utilizaron entrevistas individuales, cuyo contenido fue analizado según los conceptos del marco teórico escogido. La muestra consistió en cuatro participantes mujeres de 18 a 35 años. Los resultados indican que después de las cirugías bariátricas no sólo hubo cambios en el cuerpo físico, sino también en la significación de éste por parte de las mujeres. Junto con ello, hubo un aumento en los autocuidados, un desarrollo en la relación con el cuerpo y con el entorno social y una mejora en la autoestima. Se concluye que las operaciones bariátricas contribuyen a la unión mente cuerpo que se había fragmentado, y también que la inclusión en el período post operatorio de una terapia psicológica, que refuerce dicha integración, es valorada por las entrevistadas y vista como una forma de evitar futuras rupturas en esta integración psique- soma.

Palabras clave: Cuerpo; obesidad; significación; cirugías bariátricas.

\section{BIBLIOGRAFÍA}

1. Dolto, F. La imagen inconsciente del cuerpo. Paidós, Barcelona. 1994.

2. Aubert $N$, Haroche C. La Tirania de la visibilidad en la sociedad de hoy. Giunti Editore, Roma. 2013.

3. Ibañez, L. El problema de la obesidad en América. Rev Chil Cirugía 2007; 59:399-400. http://www.scielo.cl/scielo. php?pid=S0718- 40262007000600001\&script=sci_arttext
4. Maiz, A. Consecuencias patológicas de la obesidad: hipertensión arterial, diabetes mellitus y dislipidemia. Bol Escuela Medic 1997; 26: 18-21. http://escuela.med.puc. cl/paginas/publicaciones/boletin/html/obesidad/obesidad05.html

5. Ministerio de Salud (MINSAL). Encuesta Nacional de Salud 2009-2010. http://web.minsal.cl/portal/url/item/ bcb03d7bc28b64dfe040010165012d23.pdf

6. Rojas C; Brantes M; Miranda E; Pérez-Luco R. 2011. Descripción de manifestaciones ansiosas, depresivas $y$ autoconcepto en pacientes obesos mórbidos, sometidos a cirugía bariátrica. Rev Médica Chil. 2011. 139: 571-78. http://www.scielo.cl/pdf/rmc/v139n5/art02.pdf

7. Sociedad Española para el estudio de la obesidad (SEEDO); Consenso SEEDO 2000 para la evaluación del sobrepeso y la obesidad y el establecimiento de criterios de intervención terapéutica. Med Clin (Barc) 2000; 115: 587-97. http:// www.seedo.es/images/site/documentacionConsenso/Consenso_SEEDO_2000.pdf

8. Carrasco D, Gomez E, Staforelli A. Obesidad y Adolescencia: exploración de aspectos relacionales y emocionales. Rev Terapia Psicol. 2009 (1), 143-9. http://www.scielo.cl/scielo. php?pid=S0718-48082009000100014\&script=sci_arttext

9. Pichot P. Manual diagnóstico y estadístico de los trastornos mentales (DSM). Barcelona: Masson S.A. 1995. http:// www. mdp.edu.ar/psicologia/cendoc/archivos/DsmIV.Castellano.1995.pdf

10. Jarne, A y Colb. Psicopatología; Barcelona: Editorial UOC. 2006.

11. Bertolín, S; Leone V. Importancia de la Kinesiología dentro de un equipo interdisciplinario de salud para el tratamiento integral de la obesidad. Tesis presentada para la obtención del título de grado: Lic. Kinesiología y Fisiatría, Universidad Abierta Interamericana. Buenos Aires. 2004. http:// imgbiblio.vaneduc.edu.ar/fulltext/files/TC049536.pdf

12. Winnicott D. Escritos de Pediatría y Psicoanálisis. Paidós., Barcelona. 1945.

13. Hernández R; Fernández, C; Baptista, L. Metodología de la investigación. 2010. México: Mc Graw Hill. http:// psicologiaexperimental.files.wordpress.com/2010/03/ metodologia-de-la- investigacion.pdf 